\title{
Information Estimation on Extraction and Contents of Technological Redistribution at Steel Production
}

\author{
Kazhikenova Saule \\ Karaganda State University, Karaganda, Kazakhstan \\ Email: sauleshka555@mail.ru
}

Received October 13, 2011; revised November 20, 2011; accepted December 19, 2011

\begin{abstract}
The work suggests a formula for estimating complex indeterminancy of a group of technological operations undergoing analyses before and after their improvement, as well as technological schemes as a whole in the information units. The formula allows to estimate the complex indeterminancy of a group of technological operations undergoing analyses, as well as technological schemes as a whole, which will result in determining predictability and technological reliability of these operations.
\end{abstract}

Keywords: Entropy; Information; Hierarchical Systems; Technological Products; Technological Processes; Number of Levels

\section{Introduction}

Technical and technological modernization of industry is closely connected with the development of new and improvement of known technologies, reduction of the energy expenses on production of the product, increasing of production efficiency. The world industry, having concentrated in itself enormous a great number of enterprise on mining of ore, melting and conversion of black and non-ferrous metals, chemical and machine-building complexes, plants of precise mechanical engineering, appliance-engineering and radio electronics must receive the further development. To study the regularities of the processes of enrichment, extraction, reception, refining of metals, as well as processes, connected with the change in contents, structure and characteristic of alloys and materials in metallurgy physic-chemical and mathematical methods of the research are used. The Improvement of the technological processes with considering of the raw materials complex in structure is impossible on the only base of traditional methods of the opening the causal relationships in processes of the general technological scheme with analysis of their material and heat balances. The additional analysis of these processes is necessary on base of information entropy by Shannon; the reason for it is to integrate disembodied hitherto factors on extraction of valuable components and their contents in final products on redistribution and on technological scheme as a whole with the following use of this method for analysis and comparative estimation at chemist-metallurgical production. In this connection using as a base the information entropy by Shannon we designed a method of inte- grating disembodied hitherto factors on extraction of valuable components and their contents in final products on redistribution and on technological scheme as a whole with the following using of this method for analysis and comparative estimation of chemist-metallurgical production. As original data we used reference materials on contents and extraction of elements, published in brief guide to metallurgy of the non-ferrous metals by the authors Gudima N. B., Shein I. P. [1] and the most recent reference book under editing by M. E. Dritza [2] on the characteristics of elements in two volumes, where all the latest data from foreign reference book, monograph and scientific articles are taken into account. With the aim to conduct the comparative analysis of the competitive schemes or improving operations taken apart on united generalized criterion of complex completeness, as well as uncertainty, we shall consider usage of the formula by Shannon for determination of the information balance of the production processes by means of factors of the extraction and contents of copper. For entropy-information analysis of any object the formula by Shannon for expression of the uncertainty of the system is broadly used [3]:

$$
H=-\sum_{i=1}^{N} p_{i} \log _{2} p_{i}
$$

where pi is a probability of the finding of any uniform system element in their multitude $N ; \sum_{i=1}^{N} p_{i}=1, p_{i} \geq$ $0, i=1,2, \ldots, N$.

\section{Decision Problem}

As a characteristic of probability of finding the main sys- 
tem element it is possible to take its contents expressed in fractions of the unit. For instance, this is the contents of extracted chemical element ferric in corresponding products. The same is true for the process of the extraction of the element into one or another product, since in this case factor of the extraction is identical to probability of the transition of the given element from one state of the system into another. Both these factors - contents and extraction - can be used at equal degree for estimation of the uncertainty in product quality or technological operation. Then for the single controlled system element we use common mathematical calculations for expression of the information uncertainty as follows. If $p$ is a probability of finding of the controlled element in product or transition at extraction, then uncertainty or unexpectedness of each of these events is a reciprocal from its determined identification that is $1 / p$. In our variant of the estimation of uncertainty in the behavior of the only one system element this uncertainty is expressed by following formula:

$$
H=\log _{2} \frac{1}{p}=-\log _{2} p=-\frac{\ln p}{\ln 2}
$$

Let's define the quality of technological redistribution and distributed products on the grounds of comparing analysis of the competitive schemes on united generalized criterion of complex uncertainty and completeness of the technological scheme of steel production by domain process and direct reception ferric. Since the extraction of any component is pro rata to its contents in source material and back pro rata to its contents in product then in the first approximation extraction of ferric from terrestrial cortex in ore resources is possible to estimate on correlation:

$$
\beta_{0} \cong \frac{\alpha_{3 . \kappa .}}{\alpha_{p . M .}} \cdot 100 \%
$$

where $\beta_{0}$, a factor of the extraction at zero level of technological scheme,

$\alpha_{3 . \kappa .}$, a factor of the contents in terrestrial cortex; $\alpha_{p . M .}$, a factor of the contents in ore resources.

Since for ferric

$$
H_{k}=\sum_{i=0}^{n} H_{i}
$$

on the grounds of information formula by Shannon (2) we shall conduct entropy-information analysis of each technological redistribution for calculating the complex uncertainty and completeness of the technological scheme as a whole on example of steel production. Having received characteristic of complex uncertainty of the technological scheme it is possible by means of turned formula $p_{k}=\exp \left(-H_{k} \ln 2\right)=2^{-H_{k}}$ the parts of the unit (p.u.). (5) find corresponding to it characteristics of complex certainty of the technological scheme [4] steel production.

The results of comparative calculations on redistributions and on technological scheme of steel production by domain process as a whole are presented in Table 1, by direct reception of ferric in Table 2. The Comparing of calculating data on new model (2), (5) with practical data (Tables 1 and 2) let's illustrate graphically in coordinates in accordance with (Figure 1). At comparison of reference data on extraction and contents of the target component of technological organization of steel production by domain process (Table 1) with new model (2), (5) we revealed adequate correlation $\left(R=0,847942, t_{R}=\right.$ $6,035314>2)$ for system determination, much higher for level determination $\left(R=0.991408, t_{R}=115.8812>2\right)$. At comparison of reference data on extraction and contents of the target component of technological organization of steel production by direct reception of ferric (Table 2) with new model $(2),(5)$ we revealed identical correlation $\left(R=0.733544, t_{R}=3.176112>2\right)$ for system determination and $\left(R=0.96213, t_{R}=25.89614>2\right)$ for level determination. Calculations offered by us for information analysis of steel quality and metallurgical redistribution of the conversion of ferric already in the first approximation correlate with dynamics of raise of deterministic constituting in abstract hierarchical system.

Table 1. Information estimation on extraction and contents of technological redistribution at steel production by domain process.

\begin{tabular}{cccccccc}
\hline \multirow{2}{*}{ Technological redistributions } & \multicolumn{2}{c}{ Factors of the contents } & \multicolumn{2}{c}{ Factors of } & extraction & \multirow{2}{*}{$H_{a \beta}$} & \\
\cline { 2 - 5 } & $\alpha$ & $H_{\alpha}$ bit & $\beta$ & $H_{\beta}$ bit & & 0.0510 \\
Mining & 0.5000 & 1.0000 & 0.1020 & 3.2934 & 4.2934 & 0.5696 \\
Enrichment & 0.6550 & 0.6104 & 0.8700 & 0.2009 & 0.8113 & 0.8751 \\
Domain melting & 0.8830 & 0.1795 & 0.9910 & 0.0130 & 0.1925 & 0.9531 \\
The Smelting & 0.9550 & 0.0664 & 0.9980 & 0.0029 & 0.0693 & 0.9940 \\
Re-melting & 0.9950 & 0.0072 & 0.9990 & 0.0014 & 0.0086 & 0.9998 \\
Refining & 0.9999 & 0.0001 & 0.9999 & 0.0001 & 0.0002 & - \\
$H_{k}$ bi t & - & 1.8636 & - & 3.5117 & 5.3753 & - \\
$p_{k}$ p.u & 0.2748 & - & 0.0877 & - & - & $2.4087 \times 10^{-2}$ \\
\hline
\end{tabular}


Table 2. Information estimation on extraction and contents of technological redistribution of steel production by direct reception of ferric.

\begin{tabular}{|c|c|c|c|c|c|c|}
\hline \multirow{2}{*}{ Technological redistributions } & \multicolumn{2}{|c|}{ Factors of the contents } & \multicolumn{2}{|c|}{ Factors of extraction } & \multirow{2}{*}{$H_{\alpha \beta}$} & \multirow{2}{*}{$p_{\alpha \beta}$} \\
\hline & $\alpha$ & $H_{\alpha}$ bit & $\beta$ & $H_{\beta}$ bit & & \\
\hline Mining & 0.5000 & 1.0000 & 0.1020 & 3.2934 & 4.2934 & 0.0510 \\
\hline Enrichment & 0.7140 & 0.4860 & 0.8920 & 0.1649 & 0.6509 & 0.6368 \\
\hline Plating & 0.9800 & 0.0291 & 0.9950 & 0.0072 & 0.0363 & 0.9751 \\
\hline The Smelting & 0.9910 & 0.0130 & 0.9980 & 0.0029 & 0.0159 & 0.9890 \\
\hline Re-melting & 0.9950 & 0.0072 & 0.9990 & 0.0014 & 0.0086 & 0.9940 \\
\hline Refining & 0.9999 & 0.0001 & 0.9999 & 0.0001 & 0.0002 & 0.9998 \\
\hline$H_{k}$ bi t & - & 1.5354 & - & 3.4699 & 5.0053 & - \\
\hline$p_{k}$ p.u & 0.3449 & - & 0.0902 & - & - & $3.1131 \times 10^{-2}$ \\
\hline
\end{tabular}

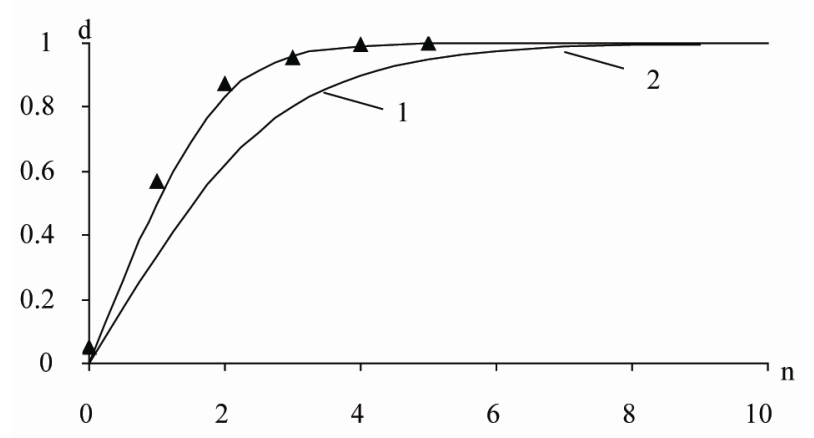

(a)

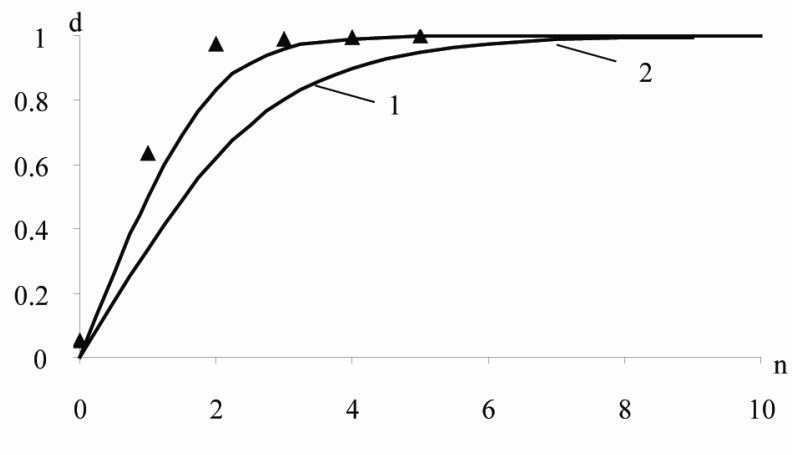

(b)

Figure 1. Dependency of the information estimation of the factors on extraction and contents from level of the technological scheme of steel production. a) Domain process; b) Direct reception of ferric; n: number of level; d- determination; 1: System determination, 2: level determination; points: practical data.

Thereby, intercoupling is set between technological factors on extraction and contents of ferric with probability of its transition and finding on every level and on technological scheme as a whole at steel production by domain process and by direct reception of ferric on the grounds of analysis of entropy-information characteristics.

With the aim to improve steel-melting production specialists from many leading metallurgical companies of the world continue to investigate ecological safe and cheaper technology of steel melting. Last years in the world steel branch they have been actively searching for profitable technology, capable to substitute the traditional process of steel production by means of domain stoves and oxygen convertor. But, as we predict the domain process of steel production will prevail on any other process of steel reception. Such is information estimation of certainty at realization of the technological schemes which can be used for comparison of their state before and after the improvement alongside with base characteristics of the complex uncertainty.

\section{Conclusions}

1) For the information analysis of quality of technolo- gical products and processes of their reception quantitative estimations of value of the information can be made only after the preliminary arrangement what exactly in each concrete case has value for considered metallurgical processes [5-9].

2) Use of the measure of certainty and uncertainty of the information allows to analyze the general mechanisms of entropy-information laws of the technological repartitions being a fundamental basis of all spontaneously proceeding processes of accumulation of the information which result in self-organizing technological processes, namely, to hierarchical systems. For multilevel hierarchical system of technological repartition it is important to describe the subordinate level as interaction of the interconnected subsystems, each of which possesses the information properties. Therefore at reception of an information estimation main attention is inverted on into-level and intra-level interactions. The considered approach, in our opinion, fully complies with the basic requirements of the system entropy-information analysis as while modeling hierarchical system of technological processes it provides integrity of its consideration due to the generaltheoretical and methodical concepts allowing to keep in sight the system as a whole entirely for the solution of a 
task at all levels of hierarchical system.

\section{REFERENCES}

[1] N. B. Gudima and I. P. Shein, "The Short Guide to Metallurgy of the Non-Ferrous Metals," Metallurgy, 1975, p. 536.

[2] M. E. Dritza, "Characteristics of Elements," 3rd Edition, Publishing House "Ore and Metals", Moscow, 2003, p. 448.

[3] C. E. Shannon, "A Mathematical Theory of Communications," Bell System Technical Journal, Vol. 27, 1948, pp. 623-656.

[4] V. P. Malyshev, S. Sh. Kazhikenova and A. Turdukozhaeva, "Qualitative and Quantitative Assessment of Technological Processes in Metallurgy of Non-Ferrous Metals," Proceedings of Higher Educational Institutions, Non-Ferrous Metallurgy, Vol. 4, 2009, pp. 22-24.
[5] S. Sh. Kazhikenova, "Information Quality Assessment of the Technological Updating," Metallurgical and Mining Industry, Vol. 4. 2009, pp. 117-123.

[6] S. Sh. Kazhikenova, "Information Analysis of the Technological Updating of the Blast Furnace Process," Steel, Vol. 9, 2009, pp. 14-16.

[7] S. Sh. Kazhikenova, "Assessment of Technological Maturity and Perfection of Production of Ferrous Metals," Problems of Ferrous Metallurgy and Materials Science, Vol. 3, 2009, pp. 91-95.

[8] V. P. Malyshev, S. Sh. Kazhikenova and A. Turdukozhaeva, "Information Technology Balance Conversion in Metallurgy," Problems of Ferrous Metallurgy and Materials Science, Vol. 1, 2010, pp. 76-83.

[9] S. Sh. Kazhikenova, "Entropy and Information Criteria for Extracting Valuable Components from Minerals," Mining Journal of Kazakhstan, Vol. 3, 2010, pp. 30-33. 